\title{
Abordagem de Avaliação da Qualidade de Sistemas Educacionais baseados em Web Semântica
}

\author{
Aparecida Maria Zem Lopes ${ }^{12}$, Seiji Isotani ${ }^{1}$ \\ ${ }^{1}$ Instituto de Ciências Matemáticas e de Computação - Universidade de São Paulo \\ Avenida Trabalhador São-carlense, 400 - Centro -13566-590 - São Carlos - SP - Brasil \\ ${ }^{2}$ Faculdade de Tecnologia de Jahu - Jaú - SP - Brasil \\ \{cida.zemegmail.com, sisotani@gmail.com\}
}

\begin{abstract}
Semantic Web-based educational systems (SWBES) are Web-based educational platforms designed to solve several problems faced by users, such as difficulties related to searching, sharing and reuse of educational resources. Ensuring the quality of these systems requires knowledge about technologies in different knowledge' areas. So far, there is no model for evaluating SWBES and, so, we have developed an approach to quality assessment that considers its multidisciplinary. This study aimed to present the approach and its evaluation, through case studies. The results showed that it is adequate, mainly for integrating the main quality criteria in a single instrument.
\end{abstract}

Resumo. Os sistemas educacionais baseados em Web Semântica (SWBES) são plataformas desenvolvidas para resolver diversos problemas enfrentados pelos usuários, relacionados à busca, compartilhamento e reuso de recursos educacionais. Garantir a qualidade desses sistemas exige conhecimento sobre tecnologias em diferentes áreas do conhecimento. Não existe, até o momento, um modelo para avaliar SWBES e, por isso, foi desenvolvida uma abordagem para avaliação de qualidade que considera sua multidisciplinaridade. Este trabalho objetiva apresentar a abordagem e sua avaliação, por meio de estudos de caso. Os resultados mostraram que é adequada, principalmente por integrar os principais critérios de qualidade num único instrumento.

\section{Introdução}

As novas tendências em tecnologias emergentes e ferramentas que dão suporte aos sistemas educacionais Web (WES - Web Educational Systems), aliadas ao papel crescente da aprendizagem social colaborativa via Web, na qual estudantes e educadores trabalham juntos em atividades, têm contribuído para que tais sistemas continuem a crescer, mudar e evoluir em todo o mundo [Adams Becker et al. 2017].

[Vladoiu e Constantinescu 2012] corroboram com essa tendência e apontam para uma ruptura no processo de formação, educação, ensino e aprendizagem, exigidas pela emergente economia do conhecimento. As autoras consideram que o conhecimento do mundo tem sido visto como um bem público que pode ser acessado e compartilhado, utilizado e reutilizado, mediado pela tecnologia, especialmente as tecnologias da informação e comunicação (TIC).

Desse modo, o processo de ensino e aprendizagem se tornou contínuo e não se limita mais aos espaços dedicados, tempos ou modalidades. A aprendizagem acontece, 
agora, ao longo da vida e envolve todos os domínios, com os usuários, as comunidades, o processo de construção social do conhecimento, as TIC do século 21 e os novos modelos de ensino aberto que compõem o núcleo dessa mudança [Vladoiu 2014], [Coutinho e Lisboa 2011].

Observa-se que os recursos de aprendizagem estão, normalmente, distribuídos nos diversos servidores educacionais. Esses recursos, criados, armazenados, atualizados por diferentes ferramentas pelos autores de conteúdo ou professores e, ainda, acessados pelos alunos que, muitas vezes, utilizam outras ferramentas, o que pode impedir a eficácia do processo de ensino e aprendizagem nesses sistemas [Devedzic 2003].

Os sistemas educacionais baseados em Web Semântica (SWBES - Semantic Web based Educational System) contribuem para resolver parte desses problemas, pois utilizam ontologias (responsáveis pela conceitualização dos recursos de aprendizagem), anotação semântica ${ }^{1}$ (baseada em ontologias) dos materiais de aprendizagem, e a entrega proativa de materiais de aprendizagem por meio de plataformas Web [Bittencourt e Costa 2009], [Devedzic 2006], [Stojanovic et al. 2001].

No entanto, para que possam ser utilizados com segurança por seus usuários, é necessário assegurar a qualidade desses sistemas. De acordo com o padrão internacional Product Quality Model ISO/IEC 25010:2011 qualidade de software pode ser definida como "o grau em que um produto de software satisfaz as necessidades explícitas e implícitas quando utilizado sob condições especificadas [ISO/IEC 25010 2008].

Avaliar a qualidade dos SWBES não é uma tarefa simples, pois são sistemas multidisciplinares que envolvem diferentes áreas do conhecimento, como a Educação, Engenharia de Software, Web Semântica, entre outras. Além disso, identificou-se uma carência de métodos e critérios de avaliação e, também, a falta de profissionais capacitados em quantidade suficiente para atender essa demanda [Lyra et al. 2003], [Roque et al. 2012].

Foi criada uma abordagem de avaliação de qualidade de SWBES com o intuito de preencher a lacuna identificada [Radulovic et al. 2015]. Nesse contexto, esse trabalho apresenta a abordagem criada e o resultado da sua avaliação, por meio de uma ferramenta Web, de acordo com os papéis e competências dos avaliadores com relação aos principais artefatos desses sistemas. A ferramenta Web, desenvolvida para automatizar a abordagem de avaliação e registrada no INPI (BR 512018 000764-3), tem por objetivo dar suporte ao complexo processo de escolha do SWBES mais adequado às necessidades de seus usuários.

\subsection{Métodos da Pesquisa}

Para o desenvolvimento da abordagem em questão, foi realizada uma pesquisa do tipo exploratória com diferentes recursos metodológicos, entre eles, um mapeamento sistemático da literatura que possibilitou obter uma visão mais ampla dos trabalhos relacionados ao domínio dos WES.

Realizou-se, também, uma revisão adhoc da literatura, com o intuito de recuperar trabalhos relacionados à avaliação de qualidade de tecnologias da Web Semântica (p. ex.,

\footnotetext{
${ }^{1}$ Anotação semântica: descreve o conteúdo de um recurso/documento por meio da associação de partes dele com os conceitos descritos na ontologia referentes a ele [Eller 2008].
} 
ontologias) e de objetos de aprendizagem (OA). Estes trabalhos são importantes para a identificação de fatores e critérios da abordagem em questão, os quais envolvem múltiplas áreas do conhecimento, tais como: Web Semântica (WS), Sistemas Educacionais Web (WES) e Engenharia de Software (ES). Além disso, utilizou-se as normas/padrões internacionais da série ISO/IEC SQuaRE 25000, e os padrões adotados pelo W3C.

Para avaliar a abordagem de avaliação de qualidade de SWBES o método utilizado foi o estudo de caso. Especialistas das áreas de gestão de TIC, Web Semântica, Informática e Educação foram convidados a avaliar o SWBES MeuTutor, por meio da ferramenta Web QualiSWBES, desenvolvida para automatizar a abordagem, sob a supervisão da pesquisadora

\section{Trabalhos relacionados}

Os trabalhos relacionados à abordagem de avaliação de qualidade em questão, utilizam critérios diversos em modelos de avaliação nas áreas multidisciplinares envolvidas.

Observou-se, por exemplo, que o modelo de [Boehm 1978] considera os fatores de qualidade adaptabilidade, interoperabilidade, reusabilidade e usabilidade apenas para o artefato software. Foram identificados modelos que avaliam a qualidade dos sistemas educacionais Web (WES), tais como [Lyra et al. 2003] e [Kelly 2008]. No entanto, apesar de considerarem as características relacionadas à educação e envolverem a aplicação das tecnologias educacionais em contextos reais de ensino e aprendizagem, esses modelos não contemplam as especificidades da Web Semântica, necessárias quando se avalia um SWBES. Além disso, avaliam apenas os artefatos OA e interface.

Já o modelo criado por [Duque-Ramos et al. 2013] avalia aspectos de confiabilidade, operacionalidade etc. das ontologias, mas não consideram suas características estruturais, tais como os casos de uso, alinhamento com outras ontologias etc. Outros modelos como os de [Bandeira 2015] e [Romano 2012] contemplam uma série de critérios de avaliação, cobrindo grande parte dos requisitos necessários para uma ontologia de qualidade, mas acabam se tornando instrumentos exaustivos aos usuários no processo de avaliação.

Assim, não se identificou um modelo consolidado para avaliação da qualidade de um SWBES, de acordo com suas características e funcionalidades esperadas/desejadas. Tais fatores de qualidade estão espalhados nesses respectivos modelos, o que torna a avaliação de um SWBES por meio deles complexo, pois envolve utilizar diversos instrumentos, cada qual com suas especificidades.

\section{Sistemas educacionais baseados em Web Semântica (SWBES)}

Os SWBES são plataformas educacionais Web desenvolvidas com o intuito de resolver diversos problemas enfrentados pelos alunos e demais usuários (professores, autores de conteúdo etc.), como dificuldades relacionadas à busca, compartilhamento e reutilização de recursos educacionais. Esses sistemas têm sido usados com frequência como motores de busca para plataformas MOOC e ITS [Konstantinos et al. 2010], [Isotani et al. 2013], [Bittencourt et al. 2008].

Um SWBES inclui: i) ontologias (requisitos importantes para definir a semântica do conteúdo, assegurar a interoperabilidade entre os sistemas de ensino etc.); ii) agentes pedagógicos (ajudam no processo de aprendizagem de diversas formas como, por 
VII Congresso Brasileiro de Informática na Educação (CBIE 2018)

Anais dos Workshops do VII Congresso Brasileiro de Informática na Educação (WCBIE 2018)

exemplo, podem avaliar semelhanças entre perfis, recomendar conteúdo educacional, compor serviços web semânticos para a educação etc.); iii) ferramentas; iv) serviços Web Semânticos (oferecem suporte às diferentes atividades educacionais e transformam coleções estáticas de informação em informação distribuída na Web com base nas tecnologias da WS, que permite que os conteúdos sejam processáveis e interpretáveis por máquina); v) ambiente Web Semântico (ambiente de interação para descobrir, navegar, selecionar e invocar recursos semanticamente anotados na Web) [Bittencourt e Costa 2009].

O processo de ensino e aprendizagem em um SWBES tem como base a consulta semântica e navegação por meio dos materiais de aprendizagem, fundamentado no background ontológico, ou seja, por meio do desenvolvimento de ontologias, anotação semântica baseada em ontologia dos materiais de aprendizagem e entrega ativa dos materiais de aprendizagem [Stojanovic et al. 2001]. A Figura 1 apresenta uma proposta de arquitetura para um SWBES.

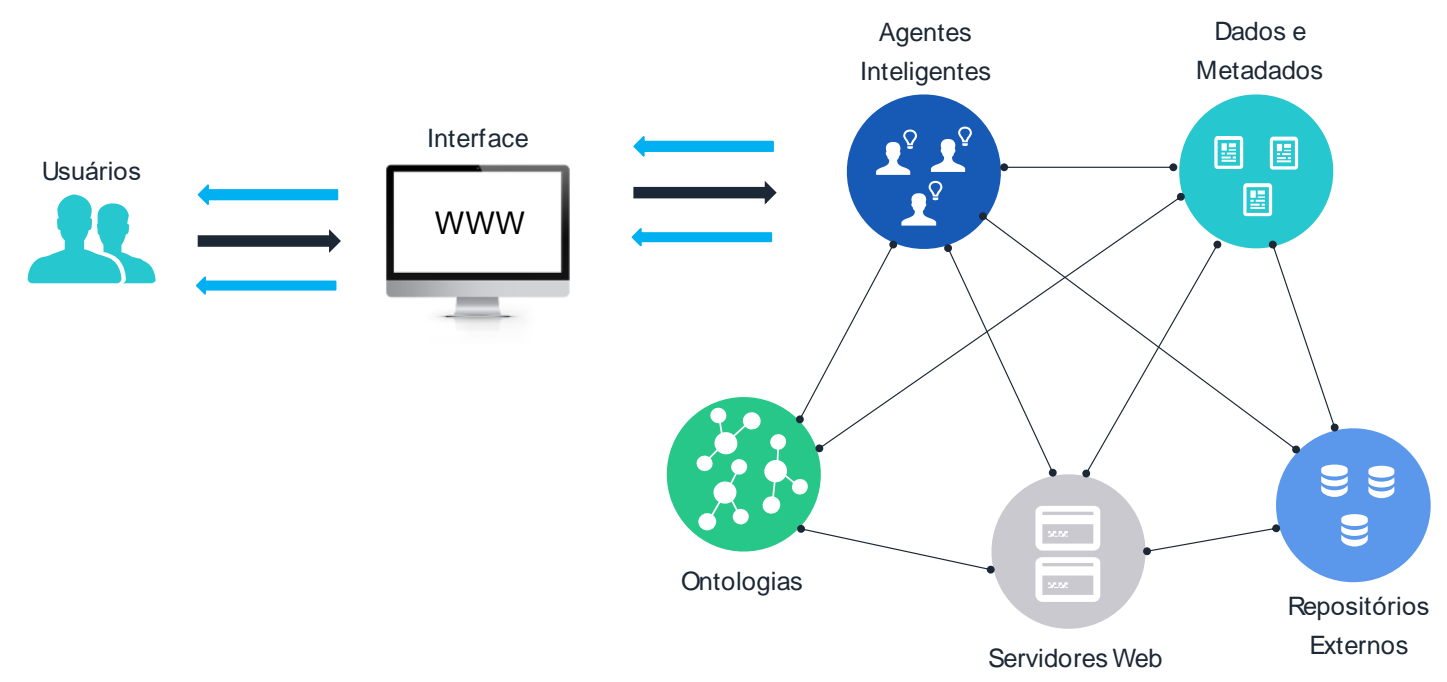

Figura 1. Arquitetura de um SWBES

\section{Abordagem de avaliação de qualidade de SWBES}

Para possibilitar melhores experiências de ensino e aprendizagem é necessário garantir a qualidade dos SWBES. No entanto, a avaliação da qualidade dos SWBES é uma tarefa complexa, uma vez que requer um amplo conhecimento sobre as tecnologias da Web Semântica, Sistemas Educacionais Web e Engenharia de Software.

Assim, a abordagem de avaliação de qualidade foi criada com o objetivo de avaliar os principais artefatos de um SWBES por meio de fatores, subfatores e critérios de qualidade definidos, a partir da competência de cada avaliador designado para a avaliação.

Os fatores de qualidade estão relacionados às funcionalidades esperadas para os SWBES, tais como adaptabilidade (aos diversos dispositivos, navegadores Web etc.), interoperabilidade com outros sistemas, reusabilidade de recursos (código fonte, modelagem, materiais de aprendizagem etc.), personalização e usabilidade. 
VII Congresso Brasileiro de Informática na Educação (CBIE 2018)

Anais dos Workshops do VII Congresso Brasileiro de Informática na Educação (WCBIE 2018)

Depois disso, decidiu-se desmembrar o SWBES em artefatos, de modo que fique mais simples avaliá-lo. Buscou-se estabelecer quais artefatos seriam mais relevantes num SWBES, que foram definidos de acordo com [Pressman 2011] e [Silva 2008]: i) Ontologia (definem um vocabulário comum para o domínio dos SWBES, composto por classes, relacionamentos, regras e instâncias); ii) Objetos de Aprendizagem (conteúdo, atividades de aprendizagem e elementos de contexto e, adicionalmente, deve conter metadados, os quais definem os atributos necessários para sua descrição completa); iii) Interface (responsável pela interação entre os usuários e o sistema); e iv) Software (qualquer elemento do sistema, como linhas de código, diagramas UML, documentos, executáveis, a própria Interface etc. A Figura 2 apresenta a sumarização dos fatores e subfatores da abordagem.
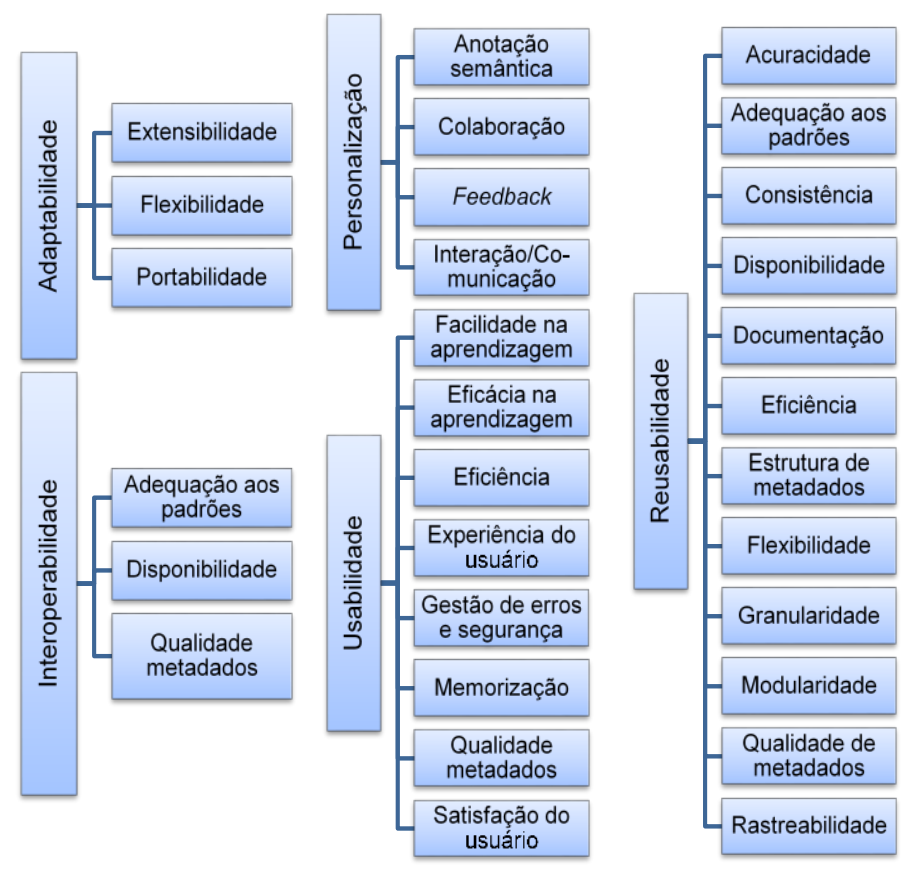

Figura 2. Visão geral dos fatores e subfatores

Quanto aos avaliadores, que serão responsáveis para avaliar os SWBES, foram estabelecidos os seguintes papéis: autor, professor, tutor, estudante, gestor, engenheiro do conhecimento/ontologias e desenvolvedor [Bittencourt e Costa 2009].

Após a definição artefatos e avaliadores, os fatores e subfatores foram subdivididos em questões, denominados critérios, que irão facilitar o processo de avaliação por parte dos avaliadores (em cada papel definido). Assim, a partir de um repositório de SWBES, os diversos profissionais e usuários (desenvolvedores, autores, professores, tutores, gestores de instituições de ensino, estudantes etc.) que precisam adquirir ou eleger um SWBES para uso, poderão realizar a escolha com base no resultado da avaliação da qualidade de cada sistema, fornecida pela abordagem.

Os avaliadores devem atribuir notas aos critérios de acordo com sua percepção com relação ao SWBES em avaliação.

Para facilitar o processo de avaliação pela abordagem, foi implementada uma ferramenta Web, denominada QualiSWBES. Por meio da ferramenta, o SWBES é cadastrado, com seus artefatos, e os avaliadores cadastrados são designados para a 
VII Congresso Brasileiro de Informática na Educação (CBIE 2018)

Anais dos Workshops do VII Congresso Brasileiro de Informática na Educação (WCBIE 2018)

avaliação e procedem a avaliação, de acordo com cada critério, subfator e fator definido na abordagem. O fluxo de avaliação da abordagem pode ser observado na Figura 3.

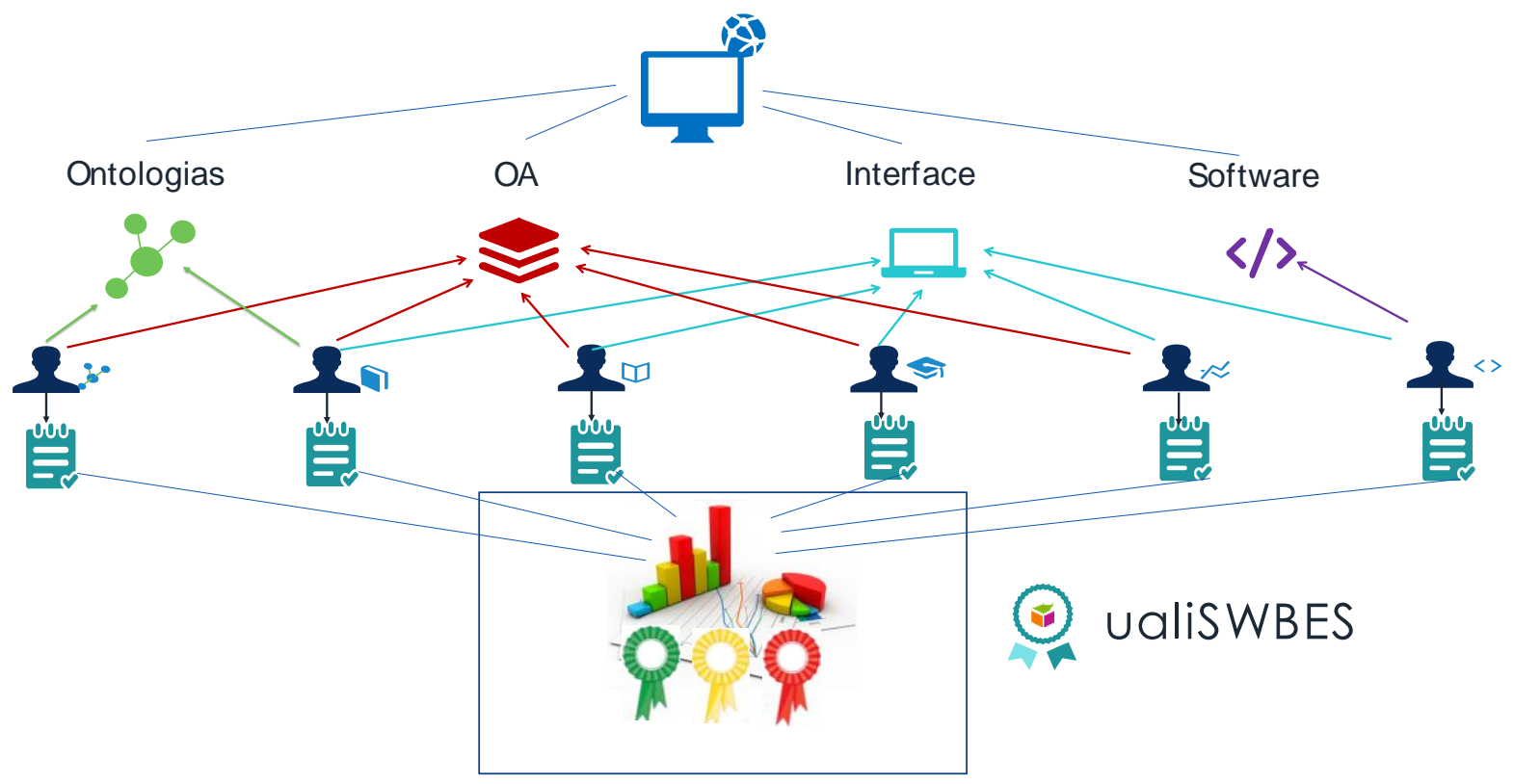

Figura 3. Modelo conceitual da abordagem

Dessa forma, os avaliadores deverão atribuir uma "nota" a cada critério que varia entre zero e cinco ( 0 e 5). Desse modo, se o artefato avaliado não atende ao critério, a nota deve ser 0 (zero) e, se atende totalmente ao critério, a nota deve ser 5 (cinco). Se o artefato atender parcialmente ao critério, o avaliador deverá atribuir uma nota entre 1 (um) e 4 (quatro). E, se porventura o avaliador não estiver apto a avaliar o critério, deve responder "não se aplica" e a nota não será computada.

Depois de concluída a avaliação por cada avaliador designado, a nota do subfator será calculada com base na média das notas atribuídas aos critérios do subfator em questão. Do mesmo modo, a nota de cada fator de qualidade será calculada com base na média de seus subfatores. Para as notas finais de cada artefato, será calculada a média das notas dos fatores de qualidade.

É importante considerar que as notas serão atribuídas pelos avaliadores em seus diversos papéis e que a nota final deve considerar a avaliação de cada avaliador para cada artefato. Em alguns casos como, por exemplo, da avaliação da ontologia e de alguns fatores que avaliam objetos de aprendizagem, será necessária a avaliação em conjunto do engenheiro de conhecimento/ontologias E do autor.

Por fim, será calculada a nota final do SWBES, que deve ser a média das notas atribuídas pelos avaliadores aos artefatos. Desse modo, o resultado da avaliação por meio da abordagem pode ser: Adequado (nota $>=4$ ), Adequado com restrições (nota entre 2.5 e 4.0) ou Inadequado (nota <2.5). Deve-se deixar claro que, a avaliação do SWBES será mais eficaz quanto maior for o domínio do avaliador sobre os artefatos do sistema e seu conhecimento na área de SWBES.

\section{Avaliação da abordagem}

Este Capítulo apresenta os resultados da aplicação da abordagem de avaliação de qualidade de SWBES, por meio da realização de estudos de caso múltiplos. A escolha de 
VII Congresso Brasileiro de Informática na Educação (CBIE 2018)

Anais dos Workshops do VII Congresso Brasileiro de Informática na Educação (WCBIE 2018)

múltiplos casos se fundamentou na literatura que aponta a vantagem em comparação aos estudos de caso único, considerando os resultados de casos múltiplos mais convincentes, e a argumentação, mais robusta [Herriott e Firestone 1983].

Os estudos de caso foram planejados com base no protocolo sugerido por [Yin 2005], que envolve o objetivo do estudo, procedimentos, questões do estudo e relatório do estudo de caso. O protocolo é apresentado a seguir.

\subsection{Objetivo}

O objetivo dos estudos de caso foi analisar a abordagem para avaliar se ela é adequada para orientar os profissionais interessados (por meio da descrição dos processos, dos artefatos, dos avaliadores, dos fatores e critérios de qualidade) a avaliarem, com sucesso, a qualidade de um SWBES, de acordo com os papéis que representam nesses sistemas, sob o ponto de vista dos especialistas em gestão de TIC, Web Semântica, Informática e Educação, no contexto dos SWBES.

\subsection{Procedimentos}

O sistema escolhido como objeto da avaliação pela abordagem foi o MeuTutor (http://meututor.com.br/), por se tratar de um SWBES em uso em diversas instituições no mercado educacional. A pesquisadora obteve permissão para utilizar os artefatos do sistema (ontologias) e, também, para acessar o sistema por meio de contas (usuário e senha) criadas exclusivamente para os estudos de caso.

Os participantes (aqui chamados de avaliadores) foram escolhidos com base na experiência acadêmica e na atuação em áreas inter-relacionadas às da abordagem, tais como gestão de TIC, Web Semântica, Informática e Educação. Além disso, possuíam visão global das áreas envolvidas e disponibilidade para participar dos estudos de caso e entrevistas.

No total, foram conduzidos oito estudos de caso (incluindo o estudo piloto). Todos os avaliadores possuíam graduação e pós-graduação (mestrado ou doutorado) na área de Ciências da Computação e ampla experiência em Informática e Educação. Entre eles, três eram especialistas no desenvolvimento de Ontologias e Web Semântica.

\subsection{Resultados e discussão}

Todos os estudos de caso realizados contaram com a participação e observação direta da pesquisadora, presencialmente ou a distância, o que contribuiu para esclarecer dúvidas pontuais durante a realização dos estudos. As entrevistas foram importantes para avaliar a abordagem, pois os avaliadores foram levados a refletir se as descrições dos processos, dos artefatos, dos papéis no sistema, dos fatores e critérios de qualidade contidos na referida abordagem, contribuem para o sucesso da avaliação da qualidade dos SWBES.

Após a análise e consolidação de todas as respostas obtidas na entrevista, a pesquisadora pôde verificar os pontos positivos e negativos apontados pelos avaliadores e que serão utilizados para nortear as atualizações e futuras versões da abordagem desenvolvida.

Foi possível observar que a abordagem foi considerada adequada para avaliar SWBES, por integrar os principais critérios de qualidade relativos às áreas da Web Semântica, Informática e Educação num mesmo instrumento de avaliação e permitir que o processo de seleção e escolha desses sistemas seja mais eficaz. 
VII Congresso Brasileiro de Informática na Educação (CBIE 2018)

Anais dos Workshops do VII Congresso Brasileiro de Informática na Educação (WCBIE 2018)

O processo de decompor o SWBES em artefatos foi bem avaliado pelos especialistas, pois possibilita que cada avaliador realize uma parte da avaliação, de acordo com sua experiência e competência, definida por um "gerente" de avaliações que consolida o resultado (facilitado pela ferramenta QualiSWBES). Desse modo, o sistema todo é avaliado por um ou mais avaliadores que avaliam um ou mais artefatos. Assim, os papéis definidos para os avaliadores foram considerados adequados e suficientes.

Por fim, alguns participantes apontaram que a abordagem tem potencial para evoluir e contribuir com o desenvolvimento de artefatos melhores, de modo que possam satisfazer as necessidades de seus usuários. Foi possível observar que, além do propósito de avaliar a abordagem, os estudos de caso permitiram identificar oportunidades, não somente de melhoria e ajustes nos fatores de qualidade estabelecidos, como também no processo de condução da avaliação.

O Quadro 1 apresenta os pontos positivos e negativos apontados pelos avaliadores na abordagem, a partir dos estudos de caso realizados.

\begin{tabular}{|c|c|}
\hline Pontos positivos & Pontos negativos \\
\hline $\begin{array}{l}\text { Integração das múltiplas áreas envolvidas num SWBES } \\
\text { num mesmo instrumento de avaliação }\end{array}$ & \multirow{2}{*}{$\begin{array}{l}\text { Quantidade elevada de critérios de qualidade } \\
\text { (questões) }\end{array}$} \\
\hline $\begin{array}{l}\text { Desmembramento do SWBES em artefatos, facilitando } \\
\text { sua avaliação }\end{array}$ & \\
\hline $\begin{array}{l}\text { Consolidação do resultado da avaliação, a partir de um } \\
\text { conjunto de avaliadores, de acordo com o papel que } \\
\text { representam no sistema }\end{array}$ & $\begin{array}{l}\text { Falta de um relatório com resultado da } \\
\text { avaliação }\end{array}$ \\
\hline $\begin{array}{l}\text { Flexibilidade proporcionada pela ferramenta } \\
\text { QualiSWBES, que permite inserir artefatos (ontologias } \\
\text { e objetos de aprendizagem) e novos critérios de } \\
\text { qualidade (questões) }\end{array}$ & \multirow{3}{*}{$\begin{array}{l}\text { Ausência de fatores de qualidade } \\
\text { relacionados à acessibilidade do SWBES }\end{array}$} \\
\hline Rapidez do processo de avaliação do SWBES & \\
\hline $\begin{array}{l}\text { Possibilidade de uso da abordagem como diretriz no } \\
\text { desenvolvimento de artefatos de melhor qualidade }\end{array}$ & \\
\hline
\end{tabular}

Como resultados adicionais dos estudos de caso, observou-se a facilidade proporcionada pela ferramenta Web QualiSWBES, bem avaliada por todos, pela facilidade de uso e simplicidade com que apresenta os critérios de qualidade, coleta as notas e simplifica o cálculo da média das notas, por critério de qualidade e artefatos.

Desse modo, espera-se que a abordagem possa contribuir para minimizar o tempo gasto pelos avaliadores ou interessados na avaliação dos SWBES, além de aumentar e garantir a eficácia da escolha realizada, por meio de uma abordagem integrada e eficiente.

\section{Considerações finais}

O processo de avaliação de qualidade de sistemas Web exige conhecimento sobre normas e requisitos relacionados às áreas de conhecimento envolvidas, além das normas técnicas relativas ao desenvolvimento de software.

Para preencher a lacuna por modelos de avaliação de qualidade de SWBES, desenvolveu-se uma abordagem para avaliação de qualidade que considera a multidisciplinaridade de tais sistemas, automatizada por uma ferramenta Web. Os fatores, subfatores e critérios de qualidade estabelecidos na abordagem, assim como os artefatos e avaliadores definidos foram fundamentais para sua eficácia. Verificou-se, a partir da 
VII Congresso Brasileiro de Informática na Educação (CBIE 2018)

Anais dos Workshops do VII Congresso Brasileiro de Informática na Educação (WCBIE 2018)

avaliação realizada, que a principal contribuição da abordagem foi a integração dos conceitos relativos às áreas envolvidas nos SWBES (Engenharia de Software, Educação e Web Semântica) num único instrumento.

Além disso, espera-se que a abordagem possa contribuir no processo de desenvolvimento de SWBES, a partir dos fatores de qualidade estabelecidos, e promover a garantia de qualidade desde o início do processo do desenvolvimento de tais sistemas.

A abordagem possibilita que os interessados (gestores de TIC, professores, autores de conteúdo, desenvolvedores de software, engenheiros do conhecimento/ontologias e até mesmo estudantes) possam avaliar a qualidade de um ou mais SWBES e escolher o mais adequado às suas necessidades, por meio de uma abordagem simples de aplicar.

\section{Referências}

[Adams Becker et al. 2017] Adams Becker, S.; Cummins, M.; Davis, A.; Freeman, A.; Hall Giesinger, C.; Ananthanarayanan, V. "NMC Horizon Report: 2017 Higher Education Edition". Austin, Texas: The New Media Consortium, 2017.

[Bandeira 2015] Bandeira, J. M. "FOCA: Uma Metodologia que utiliza princípios da Representação do Conhecimento para Avaliação de Ontologias". Dissertação (Mestrado em Modelagem Computacional do Conhecimento), Universidade Federal de Alagoas, Alagoas, Maceió, 2015, 101p.

[Bittencourt et al. 2008] Bittencourt, I. I.; Isotani, S.; Costa, E.; Mizoguchi, R. "Research directions on Semantic Web and education". Journal of ScientiaInterdisciplinary Studies in Computer Science, v. 19, n. June, p. 59-66, 2008.

[Bittencourt e Costa 2009] Bittencourt, I. I.; Costa, E. "Modelos e Ferramentas para a Construção de Sistemas Educacionais Adaptativos e Semânticos". Tese (Doutorado em Ciência da Computação), Universidade Federal de Campina Grande, Campina Grande, 2009, 246p.

[Boehm 1978] Boehm, B.; Brown, J. R., Kaspar, H.; Lipow, M.; Mcleod, G.; Merrit, M. Characteristics of software quality. North Holland, 1978.

[Coutinho e Lisboa 2011] Coutinho, C.; Lisboa, E. "Sociedade da informação, do conhecimento e da aprendizagem: desafios para educação no século XXI". Revista de Educação, v. 28, n. 1, p. 5-22, 2011.

[Devedzic 2003] Devedzic, V. B. "Key issues in next-generation web-based education". IEEE Transactions on Systems, Man and Cybernetics, Part C (Applications and Reviews), v. 33, n. 3, p. 339-349, ago. 2003, 2003.

[Devedzic 2006] Devedzic, V. "Semantic Web and Education”. Springer US: New York, 2006, 354p.

[Duque-Ramos et al. 2013] Duque-Ramos, A.; Fernandez-Breis, J. T.; Iniesta, M.; Dumontier, M.; Aranguren, M. E.; Schulz, S.; Aussenac-Gilles, N.; Stevens, R. "Evaluation of the OQuaRE framework for ontology quality". Expert Systems with Applications, v. 40, n. 7, p. 2696-2703, 2013.

[Herriott e Firestone 1983] Herriott, R. E.; Firestone, W. A. (1983) "Multisite qualitative policyresearch: Optimizing description and generalizability". Educational Researcher, 12, 14-19 
VII Congresso Brasileiro de Informática na Educação (CBIE 2018)

Anais dos Workshops do VII Congresso Brasileiro de Informática na Educação (WCBIE 2018)

[ISO/IEC 25010 2008] ISO/IEC 25010. "Software engineering-Software product Quality Requirements and Evaluation (SQuaRE) Quality model". ISO/IEC JTC1/SC7 Software and Systems Engineering Secretariat: CANADA (SCC) ISO/IEC, v. 2, p. 43, 2008.

[Isotani et al. 2013] Isotani, S. Mizoguchi, R.; Capeli, O. M.; Albuquerque, A. R. P. L.; Bittencourt, I. I.; Jaques, P. "A Semantic Web-based authoring tool to facilitate the planning of collaborative learning scenarios compliant with learning theories". Computers and Education, v. 63, p. 267-284, 2013.

[Kelly 2008] Kelly, G. "A collaborative process for evaluating new educational technologies”. Campus-Wide Information Systems, v. 25, n. 2, p. 105-113, 2008.

[Konstantinos et al. 2010] Konstantinos, M. Penelope, M.; Giannis, K.; Aglaia, L. "Semantic e-learning: Next generation of e-learning?" Lecture Notes of the Institute for Computer Sciences, Social-Informatics and Telecommunications Engineering, v. 26 LNICST, p. 295-308, 2010.

[Lyra et al. 2003] Lyra, A.; Leitão, D. "Ambiente virtual para análise de software educativo". Ambiente virtual para análise de software educativo. IX Workshop de Informática na Escola - WIE - 2003 Workshop em Informática na Educação, p. 236247, 2003.

[Pressman 2011] Pressman, R. S. "Engenharia de software”. AMGH: Porto Alegre, 2011.

[Radulovic et al. 2015] Radulovic, F.; Garcia-Castro, R.; Gomez-Perez, A. "SemQuaRE - An extension of the SQuaRE quality model for the evaluation of semantic technologies". Computer Standards and Interfaces, v. 38, n. FEBRUARY 2015, p. 101-112, 2015.

[Romano 2012] ROMANO, R. "The Quality of an Ontology: The development and demonstration of an instrument for ontology quality assessment". Thesis (Master of Information Sciences), University of Canberra, Canberra, Australia, 2012, 210p.

[Roque et al. 2012] Roque, G. O. B.; Fonseca, M. V. de A.; Campos, G. H. B. de. "A segunda geração da avaliação da qualidade de cursos A distância e os Atributos do saber fazer". v. 38, n. 2, p. 55-63, 2012.

[Silva 2008] Silva, M. T. "Autoria de Artefatos de Software”. Dissertação (Mestrado em Ciência da Computação), Fac. de Informática, PUCRS, Porto Alegre, 2008. p. 233

[Stojanovic et al. 2001] Stojanovic, L.; Staab, S.; Rudi, S. "eLearning based on the Semantic Web". WebNet2001 - World Conference on the WWW and Internet, Orlando, Florida, n. Neidl, p. 23-27, 2001.

[Vladoiu 2014] Vladoiu, M. "Towards a Quality Model for Open Courseware and Open Educational Resources". New Horizons in Web Based Learning, p. 213-220, 2014.

[Vladoiu e Constantinescu 2012] Vladoiu, M.; Constantinescu, Z. "Evaluation and Comparison of Three Open Courseware Based on Quality Criteria". Current Trends in Web Engineering, p. 204-215, 2012.

[Yin 2005] Yin, R. K. (2001) "Estudo de caso - Planejamento e Métodos". 2 ed. Porto Alegre: Bookman, 2001. 